\title{
Hypopigmentation of the Eyebrows in Frontal Fibrosing Alopecia
}

\author{
Corina Isabel Salas-Callo María Salomé Cajas-García Rodrigo Pirmez \\ Department of Dermatology, Instituto de Dermatologia Professor Rubem David Azulay, Hospital da Santa Casa da \\ Misericórdia do Rio de Janeiro, Rio de Janeiro, Brazil
}

\section{Dear Editor,}

Since the initial description of frontal fibrosing alopecia (FFA), a number of features have been incorporated to its clinical picture. Color changes such as cutaneous hypopigmentation of the alopecic band and hyperpigmentation secondary to lichen planus pigmentosus (LPPigm) are examples that the disease transcends the hair follicle $[1,2]$. In this manuscript, we describe 22 patients with FFA presenting with marked hypopigmentation of the eyebrows.

Twenty patients were women (18 postmenopausal and 2 premenopausal) and 2 men (age range 34-79 years, median age 59). All patients had skin type IV or higher and presented with bilateral hypopigmentation of the eyebrow area (Fig. 1a), which could be better appreciated on examination with a Wood's lamp (Fig. 1b). Partial or complete loss of the eyebrows was seen in all cases. Hypopigmentation along the hairline was also noted in 18 (82\%) patients (Fig. 2a). LPPigm was present in twenty cases with the diffuse pattern being the most frequent (19) followed by the reticulated pattern (1). Facial papules were observed in 13 (59\%) cases.

Hypopigmentation of the hairline has been previously described in FFA patients. Lin et al. [1] reported that FFA epidermal melanocyte counts were found to be lower than the counts seen in biopsy specimens from patients with lichen planopilaris and controls. We speculate that same mechanism could be responsible for the hypopigmentation of the eyebrow area observed clinically. An interesting observation is the sparing of this particular region in patients with LPPigm. This same phenomenon was also observed in the hairline (Fig. 2a, b). LPPigm is characterized by interface dermatitis with pigment incontinence and melanophages in the dermis which results in clinical hyperpigmentation [3]. It could be that for some unknown reason these areas are not affected by LPPigm or, alternatively, the local reduction of melanocyte counts would lead to decreased pigment incontinence in these areas. Histopathological analysis will help to clarify this point. Finally, hypopigmentation of the eyebrows is possibly better observed in patients with dark skin types, such as the ones reported herein, due to the contrast in color.

In conclusion, we describe a series of patients presenting hypopigmentation of the eyebrow area. This new observation might be in line with Lin et al. [1] findings, suggesting a pathophysiologic connection between hair follicles in FFA and the loss of epidermal melanocytes. Sparing of the hypopigmented alopecic band and eyebrow region by LPPigm is an interesting feature that needs to be better clarified and further highlights the complexity of this disease.

$\begin{aligned} & \text { karger@karger.com } \\ & \text { www.karger.com/sad }\end{aligned}$
Karger ${ }^{\prime /}$

Corina Isabel Salas-Callo

Department of Dermatology, Instituto de Dermatologia Professor Rubem David Azulay Hospital da Santa Casa da Misericórdia

Rua Santa Luzia 206, Rio de Janeiro 20020-022 (Brazil)

salas.corina@gmail.com 

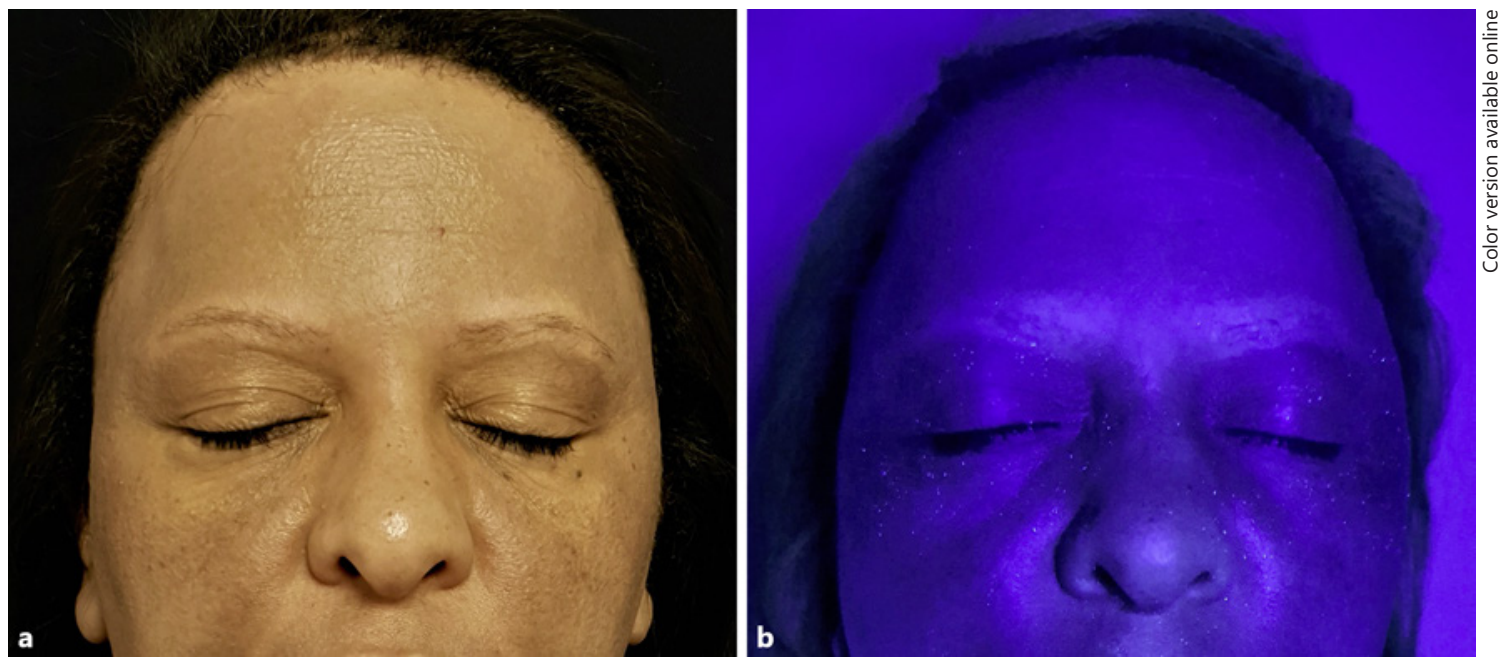

Fig. 1. Hypopigmentation of the eyebrows in FFA. a Clinical picture of a female patient with FFA showing bilateral hypopigmentation of the eyebrow area. b Same patient under Wood's light examination (365-nm ultraviolet light). FFA, frontal fibrosing alopecia.
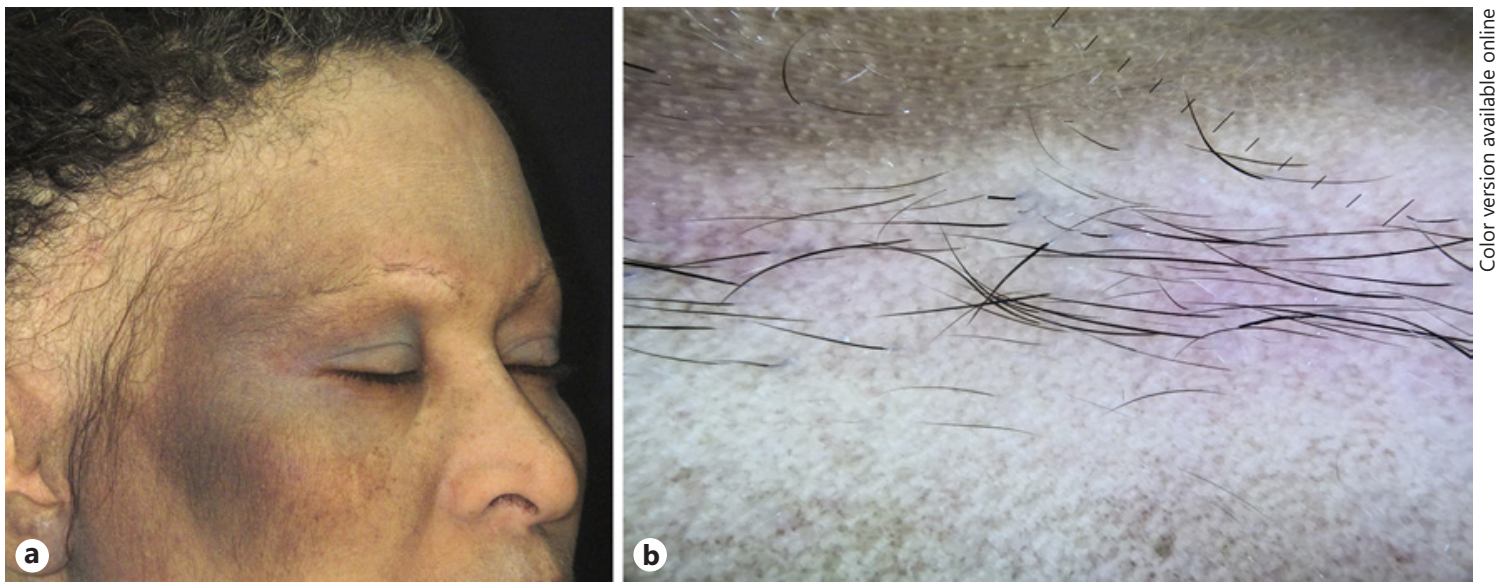

Fig. 2. FFA. a Patient with FFA and lichen planus pigmentosus presenting with hypopigmentation along the affected hairline and eyebrows. b Dermoscopic image of the eyebrow showing a clear demarcation between the hypopigmented eyebrow and the hyperpigmented adjacent skin. FFA, frontal fibrosing alopecia.

\section{Statement of Ethics}

Written consent for publication including images was obtained from the patients and research was conducted ethically in accordance with the World Medical Association Declaration of Helsinki.

\section{Conflict of Interest Statement}

The authors have no conflicts of interest to declare.

\section{Funding Sources}

There were no funding sources for this work.

\section{Author Contributions}

All authors met the criteria of the International Medical Journal Editors Committee (ICMJE) for authorship of this manuscript and approved the final manuscript. They take responsibility for the integrity of the work as a whole. 


\section{References}

Eyebrow Hypopigmentation in Frontal Fibrosing Alopecia
1 Lin J, Valdebran M, Bergfeld W, Conic RZ, Piliang M, Atanaskova Mesinkovska N. Hypopigmentation in frontal fibrosing alopecia. J Am Acad Dermatol. 2017;76(6):1184-6.

2 Pirmez R, Duque-Estrada B, Donati A, Campos-do-Carmo G, Valente NS, Romiti R, et al. Clinical and dermoscopic features of lichen planus pigmentosus in 37 patients with frontal fi- brosing alopecia. Br J Dermatol. 2016;175(6): 1387-90.

3 Saceda-Corralo D, Desai K, Pindado-Ortega C, Moreno-Arrones OM, Vañó-Galván S, Miteva M. Histological evidence for epidermal and dermal atrophy of the alopecic band in treatmentnaïve patients with Frontal Fibrosing Alopecia. J Eur Acad Dermatol Venereol. 2020 Jun 28. 